\title{
Carers continuing to care after residential care placement
}

One of the most difficult times for family carers is when the person they care for moves into a residential care home. Although they are relieved to some extent of the day-to-day tasks of caring, they lose the company of their loved one, which can be especially difficult for spouse carers. It is not always easy to obtain residential placement near to the carer's home, so carers may have to travel some distance to see the person they care for, which they often do on a daily basis. Furthermore, they lose control of the care provided, including the perceived quality of that care. They may also lose services, such as home care, or benefits, such as carer payments, that they were receiving on behalf of the person they were caring for or due to their caring responsibilities. These issues are often poorly recognized and managed in a service system that focuses more on the instrumental rather than emotional and social aspects of care for older people. It is often thought that residential care placement signifies the end of the caring role. However, qualitative research in this area highlights that family care is not relinquished when the cared for person goes into residential care and this is clearly demonstrated in the review of the qualitative literature by Afram and colleagues in this issue.

Schofield (1996) conceptualized the term "careguardianship" to recognize that carers maintain an important role in caring for the person with dementia after residential placement and that this role should be acknowledged and valued. Viewing caring in a task oriented fashion fails to acknowledge the role of the relationship and the grief associated with residential placement. She stated that, "after a bereavement through death, friends take the initiative in offering support; but when a family member moves into [residential care], there is a popular assumption that the initial reaction is relief, rather than intense sadness and pain" (Schofield, 1996; p.176). Often services that might provide support for carers, such as case management and carer support groups, withdraw upon entry to residential care; precisely when the need for emotional support is at its greatest.

This withdrawal of care reflects the often fragmented nature of aged care services, where home based services have separate funding, policy, programs, and providers to residential aged care services. Many governments are aware of the divide in service programs, however, attempts to overcome this fragmentation have had little real impact on the care of people with dementia and their carers. While goals of person- or relationship-centered care put the person with dementia and their family and carers at the center of care, the service system continues to have clear boundaries between care provided in home, residential, and hospital settings. While separate programs exist there is limited scope for providing highly valued continuity of care.

In the late 1980s, Barbara Bowers argued for a broader conception of family care that focused on the purpose of caring rather than the associated tasks (Bowers, 1987). She identified five categories of family care including anticipatory, preventive, supervisory, instrumental and protective care (Bowers, 1987). Whilst the most carer literature continues to focus on instrumental tasks, the remaining four care categories are crucial processes which can help understand the experience of carers. She identified protective care as the most important type of care and as causing the greatest stress for carers. Protective care involved trying to maintain the self-esteem of the care recipient in the context of cognitive decline. Amongst the sample of adult daughters caring for a parent in her study, protective care also aimed to maintain the role of parent and child, despite the child feeling that the roles were being reversed. Bowers also demonstrated that this form of protective or preservative care was also relevant for carers caring for a relative in residential care (Bowers, 1988).

Nolan et al. (1996) described reciprocal care as critical. In reciprocal care, the cared for person provides something of value to the carer. What they reciprocate is unlikely to be instrumental in nature but could be material or psychological. The reciprocal nature of caring is a source of carer satisfaction. Satisfaction can be derived from a sense of accomplishment and mastery; altruism; love and affection of the person they care for; feeling valued and appreciated; and helping the cared for person maintain dignity and self-respect. The greatest satisfaction is often derived from improving or maintaining the quality of life of the cared for person. Formal services that are not congruent with the carer's efforts and goals for providing 
care are likely to be rejected or experienced as a source of distress for carers. Nolan et al. (1996) also emphasize the importance of the expertise that carers develop over time through their role as carer. Residential services that do not acknowledge the expertise of the family carer may lose vital information for caring for the person with dementia and may also leave the carer feeling unheard and devalued.

A recent study that we undertook also highlighted that residential placement was not always a period of relief and respite for the family carers. Our study involved interviews with 38 family carers who looked after a relative who had been assessed as eligible for an Australian Extended Aged Care at Home Dementia (EACHD) package, and were therefore caring for someone with advanced dementia who was eligible for nursing home care (Moore, 2012). Twelve carers had placed their relative in residential care and others felt it would be inevitable. For those who were still caring for their relative at home, expectations of whether residential aged care would be required in the future were influenced by their commitment and obligation to maintain care at home for as long as possible, the person with dementia's preference to remain at home, encouragement to use residential care by other family members and health professionals, the impact of residential respite care, and the inevitability of residential placement.

For almost half of the spouse carers in our study, residential placement signified the end of long term cohabitation. This had significant implications for their well-being as cohabitation was considered a key defining element of their marital status. Carers indicated that they avoided placement as they would miss their spouse too much and two husbands who had placed their wife in residential care described difficulties resuming life without their spouse at home. One said that, "..the worst thing is, you know, when I brought her [to the facility] she just looked at me, they get these clear moments and she said 'I didn't think you would do that to me,' see. That's horrible... after 55 years of marriage. That's pretty horrible. And you have to get used to that. There's nobody in the house anymore, there's nobody sleeping next to you, you know?" Another described suicidal thoughts after not sharing his bed with his wife for the first time in 59 years. Some adult children who were caring for a parent also had strong emotional ties and commitment to maintaining care at home.

In our study, carers found that health professionals promoted residential placement. Carers perceived this as genuine concern on the part of the health professional who was generally worried about the impact that caring was having on the carer's well-being. However, in some instances health professionals strongly pressured family members to institutionalize their relative against their wishes. It appeared that in these circumstances the health professional underestimated the importance of the reciprocal relationship between the family carer and care recipient, tending to focus primarily on burden relating to physical tasks of caring. Health professionals seemed to view residential placement as a relief from caring demands and therefore a positive move to reduce burden. Whilst for some carers residential placement did reduce their workload and enabled improved sleep at night, for others the demands remained high due to concerns about the quality of care in the facility and the perceived need to visit the facility on a daily basis to assist with meals.

Many carers described how their experience of respite care shaped their views of permanent placement. For some their positive experience of residential respite gave them confidence that care would be acceptable if a more permanent arrangement was required, however, for most, their negative experiences of residential respite reinforced their negative views of the quality of residential care and strengthened their commitment to maintain care at home. While the short term nature of residential respite creates challenges to providers in developing a good understanding of the needs of the person with dementia and is therefore a barrier to person-centered care, the impact on carers of a poor experience of respite is substantial. Some carers described increased stress as a result of residential respite due to increased anxiety about their relative as well as having to deal with new health problems that occurred during respite. New problems encountered included unplanned weight loss, urinary tract infections, and pressure ulcers. Two residents had hip fractures during residential respite. Despite the goal of residential respite to reduce carer burden, carers described increased stress and an increased commitment to provide care at home.

Ryan et al. (2008) established an alternative home based program to respond to carers' dissatisfaction with residential respite care based on a relationship-centered approach in the UK. They had previously identified that the most important feature of respite identified by carers was that the person with dementia was meaningfully engaged. Respite carers were employed who understood dementia and the importance of maintaining dignity and respect for the person with dementia. One staff member cared for a person with dementia and remained their carer if they eventually moved into residential care, thereby reducing the divide between home based and residential services. 
Family members were welcome to join in respite care activities if they wanted to. The study found high satisfaction and improved quality of life amongst carers who were confident that the person with dementia was being well looked after.

In our study, in addition to wanting to fulfill the person with dementia's preference for staying at home, family carers were also influenced by other family members. Whilst some family members supported placement for the well-being of the primary carer, others tried to prevent it. Some participants were frustrated at expectations from siblings who provided minimal assistance to the primary carer to support their relative at home.

A third of carers in our study who had placed their relative in residential care identified their case manager as providing emotional support during the transition. Two carers were particularly appreciative of follow up from the case manager after the move into residential care had taken place. However, some carers also perceived that community services no longer viewed them as a carer or service user. One spouse reported that, "what happens to a carer is they seem to drop you like a hot brick once you're not a carer any longer. You know, you're just dropped out of everything. 'Ah well, we don't need you anymore.'"

The systematic review of qualitative papers by Afram and colleagues published in this issue highlights many similar issues to those that we found in our study. They found that the three main needs of carers through residential placement related to emotional concerns including shame and grief; knowledge and information about care choices and the care system; and the provision of support and counseling both through formal and informal channels. They also found that the themes were similar both prior to and after the transition, supporting the idea that the caring role does not cease after placement, and that carers' role of maintaining the self-esteem of their relative, through protective care and their role as "careguardian" continues as both a motivation and source of distress.

For many family carers looking after someone with dementia, particularly in the advanced stages of disease, the need for residential placement often becomes a necessity. The transition to residential care can be very distressing for people with dementia and their families and carers. Service providers, however, can help to reduce the level of stress that families face. Home based service providers, case managers and GPs can provide increased practical and emotional support and counseling to family carers in the decision making process as well as post residential placement when families may be experiencing grief. Residential care services also need to be aware of the impact of poor quality residential respite care and adverse outcomes during residential respite on the well-being of residents and their carers and their future perceptions of residential care. High-quality residential care is also critical in helping carers adjust and accept a transition to residential care of their relative. It is important that residential care providers acknowledge the expertise of family members regarding the care of their relative and encourage their input into care planning. Other supports such as counseling or carer support groups within the residential care environment may also assist carers to adjust to residential placement and their change of role.

At a broader systems level, the reduction in program barriers between home based and residential programs is necessary for reducing the fear, stigma, grief, and distress associated with residential placement. Having day care and respite programs co-located with residential facilities can facilitate the transition to residential respite care and permanent care. This approach can have benefits such as maintaining continuity of care staff to facilitate the development of trusting relationships; developing familiarity with the physical environment for future residential placement, and helping to break down barriers between institutional and home based forms of care.

\section{Conflict of interest}

None.

\author{
KIRSTEN J. MOORE ${ }^{1,2}$ AND BRIONY DOW ${ }^{2,3}$ \\ ${ }^{1}$ Marie Curie Palliative Care Research Department, \\ Department of Psychiatry, University College London, \\ London, UK \\ ${ }^{2}$ Health Promotion Division, National Ageing Research \\ Institute, Parkville, Victoria, Australia \\ ${ }^{3}$ Department of Psychiatry, University of Melbourne, \\ Parkville, Victoria, Australia
}

\section{References}

Bowers, B. J. (1987). Inter-generational caregiving: adult caregivers and their ageing parents. Advances in Nursing Science, 9, 20-31.

Bowers, B. J. (1988). Family perceptions of care in a nursing home. Gerontologist, 28, 361-368.

Moore, K. (2012). Informal Carers' Experiences of Extended Aged Care at Home Dementia Packages: issues of Access, Quantity and Quality of Care. Melbourne: The University of Melbourne \& the National Ageing Research Institute. 
Nolan, M., Grant, G. and Keady, J. (1996). Understanding Family care: A Multidimensional Model of Caring and Coping. Buckingham: Open University Press.

Ryan, T., Nolan, M., Reid, D. and Enderby, P. (2008). Using the Senses Framework to achieve relationship-centred dementia care services. Dementia, 7, 71-93. doi: 10.1177/1471301207085368.

Schofield, V. (1996). Careguardianship as a Phase of

Caregiving for People with Dementia (Dissertation).

Wellington: Victoria University of Wellington. 